\title{
Conséquences des sécheresses sur l'agriculture et moyens de lutte
}

\author{
C. Gaultier \\ Ministère de l'Agriculture et de la Forêt, Paris
}

\begin{abstract}
Pour n'être pas heureusement, un phénomène habituel dans notre pays, la sécheresse n'en touche pas moins assez souvent de larges fractions de son territoire. C'est ainsi que ce phénomène a été observé :

— en 1976 sur l'ensemble du territoire à l'exclusion du midi méditerranéen ;

- en 1982 dans 32 départements du sud-ouest et du massif central ;

- en 1985 dans 45 départements du sud-ouest, du massif central et de Bourgogne ;

- en 1986 dans 55 départements situés au sud d'une ligne jalonnée par La Roche-sur-Yon - Le Mans - Epinal Grenoble - Briançon.
\end{abstract}

La date d'apparition de ces sécheresses est plus ou moins tardive. Par exemple, en automne en 1985 mais dès le début de l'été en 1986. Il s'ensuit que les productions touchées varient ou encore sont différemment affectées selon la période de leur cycle de végétation durant laquelle elles ont souffert d'une alimentation en eau insuffisante. Ainsi, à la suite de la sécheresse de l'automne 1985, seule la deuxième coupe de fourrage a été perdue, la première ayant en revanche été totalement sauvegardée. Tel ne devait pas être le cas en 1986 où la sécheresse a également gravement touché la première coupe de fourrage, le phénomène étant apparu plus tôt.

Les effets de la sécheresse sur les productions agricoles dépendent également de la nature du sol, celui-ci pouvant parfois retenir en son sein des volumes d'eau suffisamment importants pour permettre la satisfaction des besoins en eau des végétaux, même en l'absence prolongée de pluies (notion de "réserve facilement utilisable ").

Toutefois, il s'agit là de situations particulières et l'agriculteur ne peut guère en général, pour pallier les effets de la sécheresse, que recourir à l'irrigation.

$\mathrm{Si}$ le coût de cette pratique culturale ainsi que des difficultés techniques liées à sa mise en œuvre peuvent apparaître dissuasifs au regard de ses promoteurs éventuels, c'est peut-être par ce qu'ils sous-estiment l'incidence sur la production agricole, de la sécheresse.

Il nous a donc paru utile de rappeler ci-après, le coût pour l'économie française des sécheresses de 1985 et de 1986, tout au moins en ce qui concerne les cultures économiquement irrigables.

\section{Consequences of droughts on agriculture and resources to combat them}

When account is taken of the extreme interannual variability of droughts, their appearance dates, duration and geographic extension, the agricultural productions affected vary and are affected differently by droughts.

Detailed tables are shown for 1985 when the drought only appeared in the Autumn and 1986 when it appeared at the beginning of the Summer.

The real amount of total losses caused to corn, sorgo, sunflower, soya and tobacco crops, can be estimated at 600 million francs in 1985 and 1800 million francs in 1986.

It is to be noted that for these two years, production, from irrigated crops was higher than the norm whereas production from dry crops was very much lower than the norm and that global harvest volume was not, therefore, that much lower, which enabled farmers who had suffered from the drought to be partially compensated by an increase in prices.

This clearly shows up the essential economic role played by irrigation. 
En effet, il ne saurait être envisagé, sauf cas d'espèce, d'irriguer des productions telles les fourrages ou les céréales à paille. Leur prix de vente, ainsi que d'autres contraintes économiques (quotas laitiers notamment) ne sauraient justifier les investissements exigés. De plus, concernant les céréales à paille, l'époque de leur récolte leur permet presque toujours d'échapper aux effets de la sécheresse. En revanche, des productions comme le maïs, le sorgho, le tournesol, le. soja et le tabac tirent un grand bénéfice de l'irrigation en période de sécheresse. C'est ce que met en relief les tableaux ci-joints relatifs aux sécheresses de 1985 et de 1986. Ces tableaux qui ont été établis à partir de données recueillies auprès du fonds de garantie des calamités agricoles, ne font état que du montant des indemnités versées aux agriculteurs sinistrés, soit moins de $30 \%$ en moyenne du montant des dommages indemnisables.

Ces dommages indemnisables sont par ailleurs sensiblement inférieurs à la réalité, puisque le fonds de garantie des calamités agricoles n'indemnise que les pertes de récolte qui répondent à la double condition de :

- représenter au moins $27 \%$ de la ou des productions sinistrées ;

- représenter au moins $14 \%$ du produit brut de l'exploitation affectée par le sinistre. Le montant des pertes causées à l'ensemble des cultures de maïs, sorgho, tournesol, soja et tabac par la sécheresse peut dans ces conditions être estimé à $600 \mathrm{MF}$ en $1985,1800 \mathrm{MF}$ en 1986.

Il est également intéressant d'observer qu'à l'intérieur d'un même département les rendements des cultures irriguées étaient en 1985 et 1986 supérieurs à la normale de ces cultures, alors que les rendements des cultures en sec étaient eux très inférieurs à la normale de ces dernières cultures.

Sans être exhaustifs les exemples ci-après, relatifs à la sécheresse de 1986 illustrent cette constatation.

\section{GIRONDE}

\begin{tabular}{|l|c|c|c|c|c|c|c|}
\hline Culture & $\begin{array}{c}\text { Surface } \\
\text { totale }\end{array}$ & $\begin{array}{c}\text { Surface } \\
\text { irriguée }\end{array}$ & $\begin{array}{c}\text { Rendement } \\
\text { moyen en } \\
\text { irrigué }\end{array}$ & $\begin{array}{c}\text { Rendement } \\
1986 \text { en } \\
\text { irrigué }\end{array}$ & $\begin{array}{c}\text { Surface } \\
\text { en sec }\end{array}$ & $\begin{array}{c}\text { Rendement } \\
\text { moyen en } \\
\text { sec }\end{array}$ & $\begin{array}{c}\text { Rendement } \\
1986 \\
\text { en sec }\end{array}$ \\
\hline Maïs & $42000 \mathrm{ha}$ & $23000 \mathrm{ha}$ & $85 \mathrm{qx} / \mathrm{ha}$ & $110 \mathrm{qx} / \mathrm{ha}$ & $19000 \mathrm{ha}$ & $55 \mathrm{qx} / \mathrm{ha}$ & $27 \mathrm{qx} / \mathrm{ha}$ \\
\hline
\end{tabular}

VIENNE (sud du département)

\begin{tabular}{|l|c|c|c|c|c|c|c|}
\hline Cultures & $\begin{array}{c}\text { Surface } \\
\text { totale }\end{array}$ & $\begin{array}{c}\text { Surface } \\
\text { irriguée }\end{array}$ & $\begin{array}{c}\text { Rendement } \\
\text { moyen en } \\
\text { irrigué }\end{array}$ & $\begin{array}{c}\text { Rendement } \\
1986 \text { en } \\
\text { irrigué }\end{array}$ & $\begin{array}{c}\text { Surface } \\
\text { en sec }\end{array}$ & $\begin{array}{c}\text { Rendement } \\
\text { moyen en } \\
\text { sec }\end{array}$ & $\begin{array}{c}\text { Rendement } \\
1986 \\
\text { en sec }\end{array}$ \\
\hline $\begin{array}{l}\text { Maïs } \\
\text { Tournesol }\end{array}$ & $\begin{array}{r}21000 \mathrm{ha} \\
65000 \mathrm{ha}\end{array}$ & $\begin{array}{r}7000 \mathrm{ha} \\
17000 \mathrm{ha}\end{array}$ & $\begin{array}{c}75 \mathrm{qx} / \mathrm{ha} \\
25 \mathrm{qx} / \mathrm{ha}\end{array}$ & $\begin{array}{r}100 \mathrm{qx} / \mathrm{ha} \\
28 \mathrm{qx} / \mathrm{ha}\end{array}$ & $\begin{array}{r}14000 \mathrm{ha} \\
48000 \mathrm{ha}\end{array}$ & $\begin{array}{r}509 \mathrm{qx} / \mathrm{ha} \\
20 \mathrm{qx} / \mathrm{ha}\end{array}$ & $\begin{array}{c}35 \mathrm{qx} / \mathrm{ha} \\
17 \mathrm{qx} / \mathrm{ha}\end{array}$ \\
\hline
\end{tabular}

\section{LANDES}

\begin{tabular}{|l|c|c|c|c|c|c|c|}
\hline Culture & $\begin{array}{c}\text { Surface } \\
\text { totale }\end{array}$ & $\begin{array}{c}\text { Surface } \\
\text { irriguée }\end{array}$ & $\begin{array}{c}\text { Rendement } \\
\text { moyen en } \\
\text { irrigué }\end{array}$ & $\begin{array}{c}\text { Rendement } \\
1986 \text { en } \\
\text { irrigué }\end{array}$ & $\begin{array}{c}\text { Surface } \\
\text { en sec }\end{array}$ & $\begin{array}{c}\text { Rendement } \\
\text { moyen en } \\
\text { sec }\end{array}$ & $\begin{array}{c}\text { Rendement } \\
1986 \\
\text { en sec }\end{array}$ \\
\hline Maïs & $135000 \mathrm{ha}$ & $113000 \mathrm{ha}$ & $87 \mathrm{qx} / \mathrm{ha}$ & $90 \mathrm{qx} / \mathrm{ha}$ & $22000 \mathrm{ha}$ & $64 \mathrm{qx} / \mathrm{ha}$ & $30 \mathrm{qx} / \mathrm{ha}$ \\
\hline
\end{tabular}

ARIËGE

\begin{tabular}{|c|c|c|c|c|c|c|c|}
\hline Culture & $\begin{array}{c}\text { Surface } \\
\text { totale }\end{array}$ & $\begin{array}{l}\text { Surface } \\
\text { irriguée }\end{array}$ & $\begin{array}{c}\text { Rendement } \\
\text { moyen en } \\
\text { irrigué }\end{array}$ & $\begin{array}{c}\text { Rendement } \\
1986 \text { en } \\
\text { irrigué }\end{array}$ & $\begin{array}{l}\text { Surface } \\
\text { en sec }\end{array}$ & $\begin{array}{l}\text { Rendement } \\
\text { moyen en } \\
\text { sec }\end{array}$ & $\begin{array}{c}\text { Rendement } \\
1986 \\
\text { en sec }\end{array}$ \\
\hline Maïs & $12500 \mathrm{ha}$ & 6000 ha & $70 \mathrm{qx} / \mathrm{ha}$ & 84 qx/ha & 6500 ha & $38 \mathrm{qx} / \mathrm{ha}$ & $25 \mathrm{qx} / \mathrm{ha}$ \\
\hline Sorgho & 400 ha & $150 \mathrm{ha}$ & $60 \mathrm{qx} / \mathrm{ha}$ & $85 \mathrm{qx} / \mathrm{ha}$ & 250 ha & $40 \mathrm{qx} / \mathrm{ha}$ & $21 \mathrm{qx} / \mathrm{ha}$ \\
\hline Tournesol & 2100 ha & 840 ha & $20 \mathrm{qx} / \mathrm{ha}$ & $28 \mathrm{q} x / \mathrm{ha}$ & 1260 ha & $16 \mathrm{qx} / \mathrm{ha}$ & $10 \mathrm{qx} / \mathrm{ha}$ \\
\hline Soja & 2350 ha & 1550 ha & $25 \mathrm{qx} / \mathrm{ha}$ & $27 \mathrm{q} \times / \mathrm{ha}$ & 800 ha & $19 \mathrm{qx} / \mathrm{ha}$ & \\
\hline
\end{tabular}


Ceci explique que malgré l'importance des pertes subies par de très nombreux agriculteurs, le volume global de la récolte de maïs n'ait pas fortement fléchi et qu'en conséquence les cours de cette céréale ne se soient pas relevés de façon significative. Les agriculteurs touchés par le sinistre n'ont donc pu bénéficier d'une compensation de leurs pertes par le jeu d'une majoration des prix de commercialisation de leurs produits.

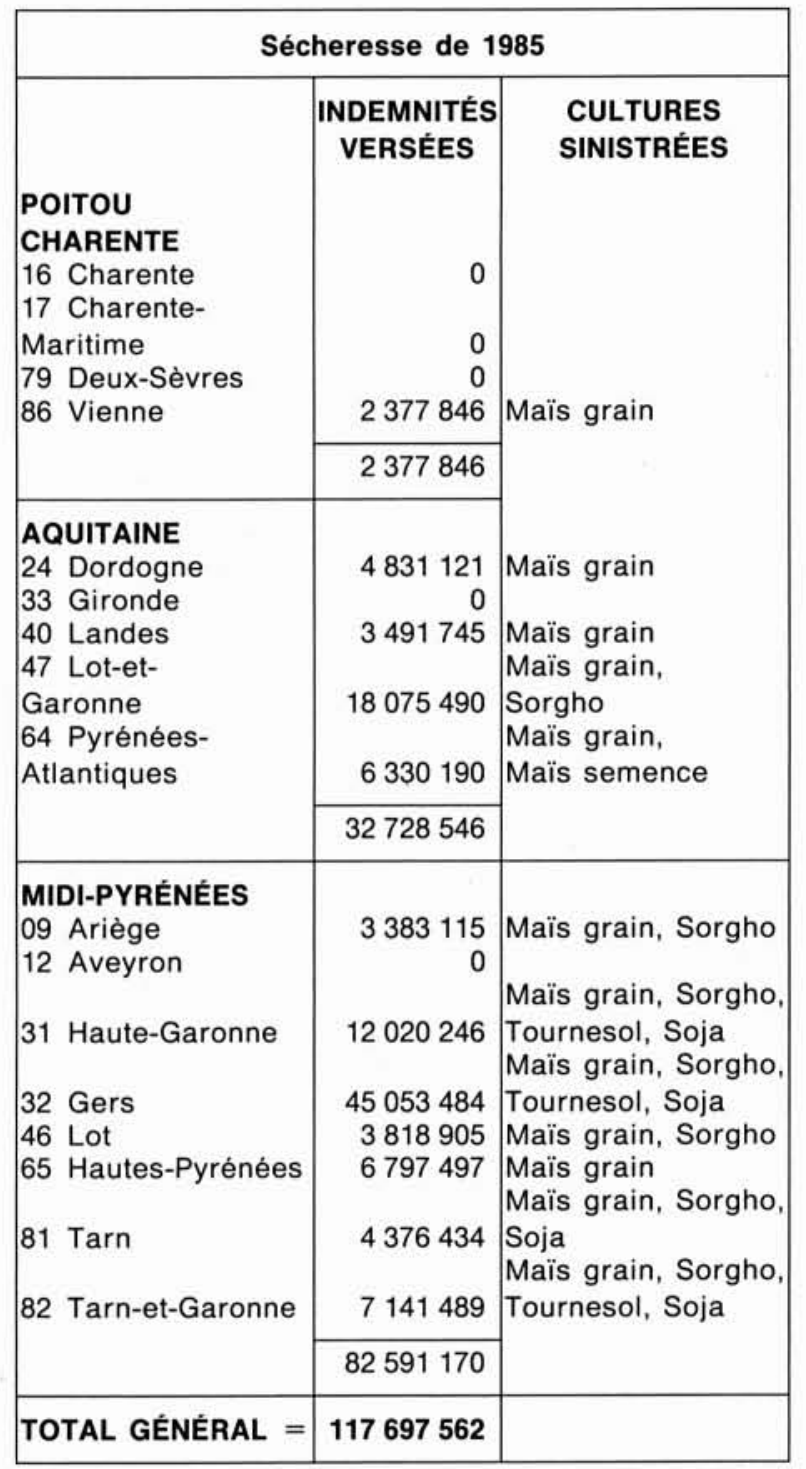

\begin{tabular}{|l|c|c|}
\hline \multicolumn{3}{|c|}{ Sécheresse de 1986} \\
\hline DÉPARTEMENTS & $\begin{array}{c}\text { INDEMNITÉS } \\
\text { VERSÉES }\end{array}$ & $\begin{array}{c}\text { CULTURES } \\
\text { SINISTRÉES }\end{array}$ \\
DORDOGNE & 130826 & Tabac \\
LOT-ET-GARONNE & 70714 & Tabac \\
ARIËGE & 30354 & Tabac \\
GERS & 632695 & Tabac \\
LOT & 157855 & Tabac \\
TARN & 389756 & Tabac \\
& 1412200 & \\
\cline { 2 - 2 } & & \\
\hline
\end{tabular}

Cette situation nouvelle, que renforce par ailleurs les facilités de circulation accordées aux produits agricoles à l'intérieur de la CEE ne peut que rendre plus fragile l'équilibre économique des exploitations agricoles du sud-ouest qui ne pratiquent pas encore l'irrigation sur leurs cultures de maïs, sorgho, tournesol, soja et tabac.

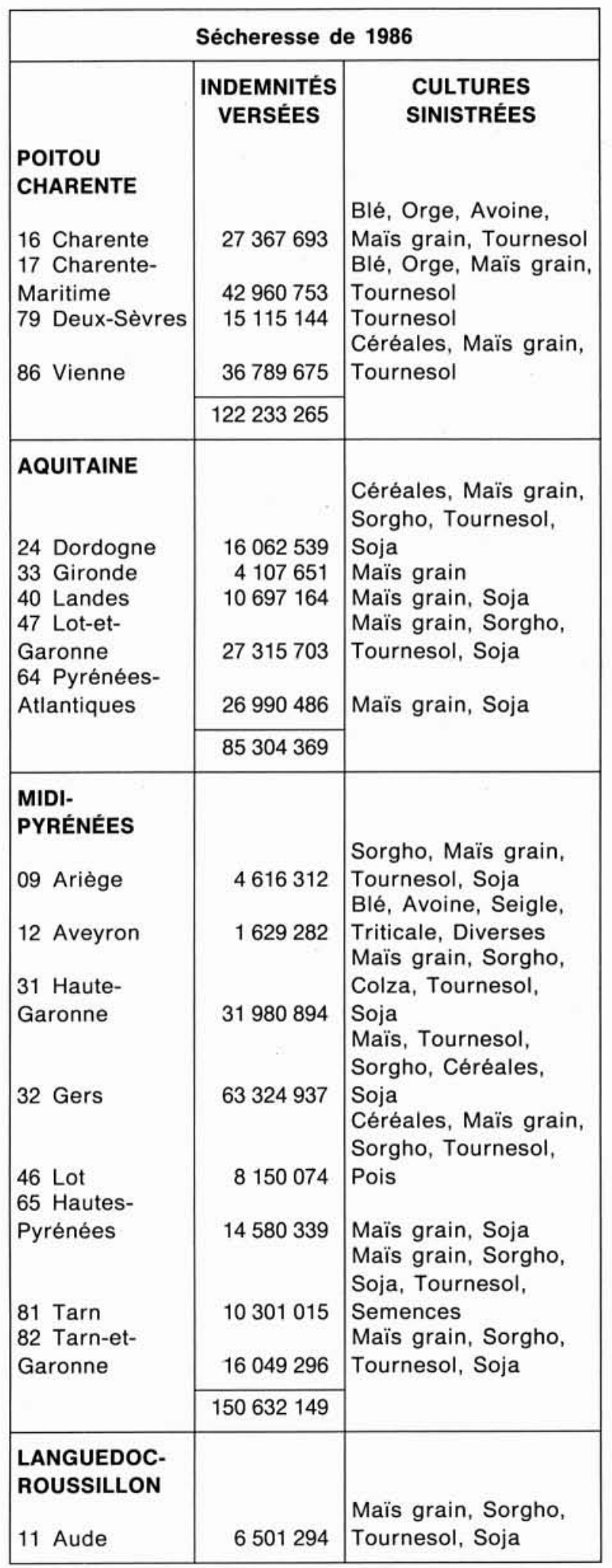

